\title{
Review
}

\section{LCA Practices of Plastics and Their Recycling: A Critical Review}

\author{
Timothy M. Kousemaker ${ }^{1}$, Gerald H. Jonker ${ }^{2}$ and Antonis I. Vakis ${ }^{3, *(\mathbb{D}}$ \\ 1 CMME/PT, ENTEG, FSE, University of Groningen, Nijenborgh 4, 9747 AG Groningen, The Netherlands; \\ t.m.kousemaker@rug.nl \\ 2 Design Group, FSE, University of Groningen, Nijenborgh 4, 9747 AG Groningen, The Netherlands; \\ g.h.jonker@rug.nl \\ 3 CMME, ENTEG, FSE, University of Groningen, Nijenborgh 4, 9747 AG Groningen, The Netherlands \\ * Correspondence: a.vakis@rug.nl
}

check for updates

Citation: Kousemaker, T.M.; Jonker, G.H.; Vakis, A.I. LCA Practices of Plastics and Their Recycling: A Critical Review. Appl. Sci. 2021, 11, 3305. https://doi.org/10.3390/app11083305

Academic Editors:

Francesco Ciardelli and

Francesco Picchioni

Received: 1 March 2021

Accepted: 31 March 2021

Published: 7 April 2021

Publisher's Note: MDPI stays neutral with regard to jurisdictional claims in published maps and institutional affiliations.

Copyright: (c) 2021 by the authors. Licensee MDPI, Basel, Switzerland. This article is an open access article distributed under the terms and conditions of the Creative Commons Attribution (CC BY) license (https:// creativecommons.org/licenses/by/ $4.0 /)$.

\begin{abstract}
In a bid to help address the environmental footprints associated with products and services, life cycle assessment (LCA) applications have become increasingly popular throughout the years. This review summarizes some important methodological developments in recent years, such as the advent of dynamic LCA, as well as highlighting recent LCA applications in the context of plastics/recycling with a focus on their methodological choices. Furthermore, this review aims to offer a set of possible research lines to improve the gap between LCA and decision-making (policy). It was found that the majority of reviewed papers are mostly conservative in their methodological practice, employing mostly static analyses and making little use of other methods. In order to bridge the gap between LCA and policy, it is suggested to broaden system boundaries through the integration of dynamic modelling methods, incorporating interactions between fore- and background systems, and including behavioral components where relevant. In addition, advanced sampling routines to further explore and assess the policy space are recommended. This is of paramount importance when dealing with recycling processes as the molecules/polymers constituting the output of those processes have to be benchmarked in terms of costs and, crucially, their sustainability character against virgin ones.
\end{abstract}

Keywords: LCA; dynamic LCA; recycling; policy; multi method; dynamic modeling

\section{Introduction}

The 20th century marked an era in which advances in polymer science and technology allowed humankind to explore and use many new functional chemical products such as plastics and rubbers. Since then, many polymeric products have been responsible for a marked improvement of (engineering) materials. Examples include, amongst others, polyethylene terephthalate (PET) (often used in consumer bottles) and polytetrafluoroethylene (PTFE) for non-stick coatings in cooking pans, among other materials. [1,2] However, back in those pioneering days, few could have foreseen that by 2025 , the oceans might contain as much as 250 million metric tons of plastic [3]. The scale and magnitude of these environmental problems are now clear and must be reduced with urgency. Obviously, methods to identify the range of possible environmental impacts of (chemical) products is an important step to prevent such problems being exacerbated or reemerging in the future.

The life cycle assessment (LCA) is seen as one of the methods to estimate and quantify the environmental impact of products, systems, and services by mapping mass and energy balances [4]. The method has enjoyed widespread recognition and use, with a surge of academic output throughout the past few decades. As a result of the many applications and debates that have arisen, modifications and extensions have been proposed by various authors [5,6]. One of those includes the use of temporal analysis, which is often referred to as dynamic LCA (DLCA). Advantages of this approach include the ability to assess the dynamics of systems, as conventional LCA often conveniently assumes linearity and a steady state [7]. 
This review intends to provide an overview and discussion of (modern) methods used to perform DLCA, as well as some recent applications in the context of plastics and recycling, to assess the current methodological state-of-the-art procedures in the field. An attempt is made to identify areas of improvements to further advance the use of DLCA methods in product design and end-of-life applications such as recycling in order to improve the connection between model and policy. To this end, first an overview of LCA and its modelling approaches is briefly described and discussed. This is followed by an overview of DLCA and DLCA applications in plastics and recycling to summarize the main methodological choices made in recent contributions. Finally, methodological issues are discussed concerning DLCA and potential research lines are proposed to further enhance the practice of using such methods to guide policy.

\section{The Methodological Foundations and Advances in Life Cycle Assessment}

The first foundations for LCA were laid back in the US in the 1960s, as scientific communities around the world sought to capture the complexity of environmental issues. Most notably, the first formalized attempt was made by Harry E. Teasly Jr., who during that time was working for the Coca Cola company [8]. It took a while before the approach moved away from its initial focus on energy until a broader sense of its use along with standardization emerged in the 1990s [9]. These days, LCA has been standardized in International Standards Organization (ISO) standards 14040 and $14044[10,11]$. The LCA consists of 4 distinct phases, which can be seen below in Figure 1, and finds its applications in a variety of settings (e.g., new product development or policy).

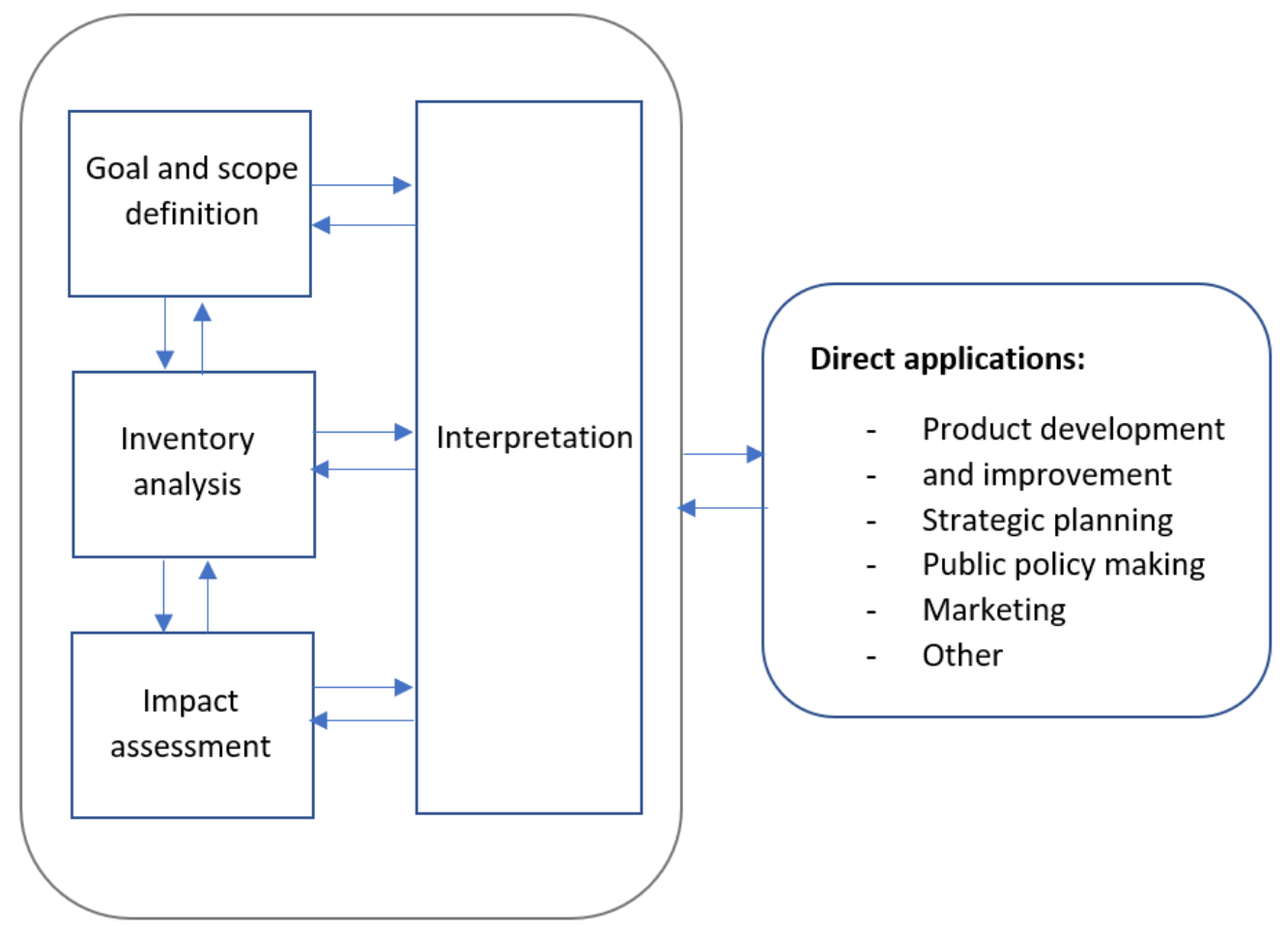

Figure 1. Life cycle assessment (LCA) phases according to ISO standards [11].

\subsection{The Four Phases According to ISO 14040/14044}

\subsubsection{Goal and Scope Definition}

According to the ISO 14044 norms, the goal and scope of an LCA study must be clearly defined, and any changes in goal and scope during the project must be documented. After selection of the product system and a quantified description of a product's function (functional unit), flow charts are often used to map the processes that describe the product system. The generic aim is a linear system definition [12]. It is recognized at this stage that 
it is important for the modeler to be aware of the goal of the analysis, as this determines whether the LCA is either attributional or consequential [13] — which are both explained in the section covering LCA approaches.

\subsubsection{Inventory Analysis (LCI)}

This phase in essence constitutes a simplified linear systems analysis which accounts for all flows into and out of the system, depending on the chosen functional unit. It is one of the most comprehensive phases in the LCA study and requires the modeler to make various choices with respect to system boundaries, how to distribute and weigh inputs and outputs, and whether or not to perform a closed or open loop analysis [12]. The obtained model is typically static. As a consequence of the linear model, the results scale linearly with the functional unit [13].

\subsubsection{Impact Assessment (LCIA)}

In this phase, the analyst aims to quantify the interaction of the product system with the environment. To this end, impact categories, indicators and characterization models need to be selected, and coupling the results from the inventory analysis to the chosen impact categories $[12,14]$.

\subsubsection{Interpretation}

In this phase, the findings of the inventory analysis and impact assessment are jointly evaluated and should yield results consistent with the defined goal and scope, allowing the analyst to draw conclusions and provide recommendations. The steps consist of [12]:

1. identifying significant issues based on the preceding inventory and impact assessment phases;

2. a thorough evaluation to check whether the work is complete and consistent, as well as sensitivity and or scenario analyses;

3. a set of recommendations, conclusions and limitations.

Mathematically, the structure of such an LCA model can be resembled in matrix notation as follows:

$$
s=A^{-1} f
$$

where $s$ represents the scaling vector, $A$ the technology matrix, and $f$ the final demand vector. The scaling vector indicates how much each process output is used with respect to the functional unit, while the technology matrix contains rows indicating the products (e.g., $\mathrm{kg}$ of plastic) and columns representing the unit processes (production of plastic) [15].

\subsection{Types of $L C A$}

While ISO 14044 documentation is clear about the standards that should be adhered to in LCA analyses, modelers face various challenges. In particular, the inventory analysis (LCI) and impact assessment (LCIA) are subject to a great variety of methodological considerations depending on the chosen modeling approach. One can distinguish between various streams of modelling approaches and corresponding accounting methods within LCA. This section provides an overview and characterization of major developments in LCA approaches.

\subsubsection{Attributional vs. Consequential LCA}

The differences between attributional and consequential LCA approaches in modelling become clear in the life cycle inventory analysis phase, where, in case of an attributional LCA (aLCA), modelers attribute inputs and outputs to the functional unit of the product within a chosen temporal window by linking the unit processes of the system according to a normative rule (e.g., based on physical processes or geographical boundaries [16]).

When a modeler uses consequential LCA (CLCA), the analysis is geared towards describing how changes and decisions in the product system relate to the (environmental) 
flows of interest [17-19]. In essence, an aLCA assumes there is no interaction between decisions made within the chosen system and the outside world, whereas cLCA does [20,21]. As a result, cLCA tends to yield more complex models which investigate impacts over a wider range of temporal and spatial horizons, with models that are not necessarily static and linear, as is the case with aLCA [20,22], although it should be noted that such models are still far from being dynamic.

Another point of differentiation in LCA approaches refers to how the study looks at the time component of the data and decisions of the product system. In some cases, the terms 'prospective' and 'retrospective' are used to respectively refer to studies that are concerned with future situations or situations that occurred in the past [19]. It is important to note that these terms are sometimes used loosely. For instance, [23] used the term 'prospective' in a broader sense and used it to reflect an LCA that assesses consequences of decisions made in the past, but evaluated at a later point in time. At the same time, prospective LCAs are also coined 'ex-ante' or 'anticipatory' by others [24]; such applications will be discussed later on.

The choice for a prospective or retrospective study usually has implications for the system boundaries. Typically, these are expanded when a prospective LCA is performed to include activities and corresponding emissions resulting from substitution due to the new product system. In addition, the difference in the goal of the study is also reflected in the methodological choices made in the LCI, as this phase is concerned with quantifying the relevant flows. In particular, compiling the data can become problematic as the static nature of the model requires an estimate of the data to be used within the chosen time horizon. Questions might arise whether or not to average data within a time frame, or to use marginal data. In case of a retrospective LCA, the latter is not used [23,25]. This might be somewhat confusing, as the case of marginal vs. average data is typically also a main difference between CLCA and aLCA [26]; cLCA tends to use marginal data in order to reflect on what happens when changes are made within the product system. Others [23] have discussed the notion that retrospective LCAs are useful to learn about life cycles and subsystems and avoid undesirable impacts, while prospective LCAs are suitable for decision-making by stating that both methods can be used for decision-making and learning purposes.

There are some strong limitations to these types of LCA. For instance, [27] extensively discussed inherent weaknesses in data quality, lack of consideration of temporal and spatial effects, the problem with allocating flows to product systems by means of Input-Output (I/O) tables, and uncertainty.

\subsubsection{Allocation Methods: Accounting for the Environmental Impacts}

In the context of recycling and end-of-life (i.e., products entering new lifecycles or functions), various methods exist to allocate the emissions of product systems when they face such multifunctionality. Recently, [28] proposed a framework to solve the allocation problem that occurs when product systems are faced with multifunctionality, to avoid inconsistent comparisons between methods and product systems. These include the cut-off approach, system expansion, partitioning, end-of-life recycling method, waste mining method, and 50/50 method. The choice for an aLCA or cLCA impacts how to credit environmental burdens. Table 1 summarizes the suitability of the following accounting methods in the context of aLCA and CLCA [28]:

(a) In the system expansion method, the co-functions that arise due to the new life cycle that consumes the recycled product are incorporated in the functional unit [29]. This method ensures proper accounting of the burdens and credits. In the consequential approach it leads to substitution as conventional products and their impacts are avoided.

(b) In the partitioning method, inputs and outputs of the system are allocated to functions according to a set of criteria, thereby distributing the burden and impact among the primary and recycled/co-products. 
(c) The cut-off approach refers to a situation in which the modeler or analyst excludes the second function of the recycled product and only includes the products and processes that are directly related to the functional unit. This means that waste management and recycling functions are attributed to the second life cycle of the product $[30,31]$.

(d) The waste mining method imposes the burdens associated with recycling on the recycled material input of the system and no benefits are attributed to the production of recycled material.

(e) The 50/50 method equally distributes credits and burdens between the different life cycles as a sort of compromise and could be seen as a quick and dirty method in that respect, although it is recommended by some [32].

(f) The end-of-life recycling method assigns benefits to the production of recycled materials since it mitigates the production of primary material. A quality correction factor is used to account for the displacement of primary material as a result of the recycled material use.

Table 1. Overview of accounting methods per LCA type, based on [28].

\begin{tabular}{ccc}
\hline Accounting Method & Attributional LCA & Consequential LCA \\
\hline System Expansion & $\mathrm{X}$ & $\mathrm{X}^{*}$ \\
Partitioning & $\mathrm{X}$ & \\
Cut-off Approach & $\mathrm{X}$ & $\mathrm{X}$ \\
Waste Mining & & $\mathrm{X}$ \\
$50 / 50$ & & $\mathrm{X}$ \\
End-of-Life Recycling & & \\
\hline
\end{tabular}

* In the consequential approach, system expansion leads to substitution. Modelers can choose from the substitution methods 'Waste Mining', ‘50/50', and 'End-of-Life Recycling'.

The main problem with all these methods is the intrinsic assumption of a linear, static system, as well as the desire to try and allocate everything to a single (or multiple) functional unit(s), instead of calculating total system outputs throughout its lifetime, taking into account the dynamics of the system. When there are (multiple) loops and multifunctionality, it matters for the modeler which method is chosen to allocate the environmental burdens of a product as the model will yield different outcomes, but for total system emission it does not matter; the outcome remains the same, no matter how one allocates emissions to products and processes. Yet the conclusion of an LCA study is very much dependent on the methodological assumptions and choices concerning these allocation methods. This poses a serious risk of undermining the external validity of such studies as their conclusions are strongly determined by the accounting method that was selected, with risks of being skewed and leading to wrong policy advice. In a policy context, [33] described the major differences that can occur depending on the choice of allocation method. Other authors also found that these methodological choices can skew results, especially if the uncertainty space of the model is not investigated rigorously [34].

It might be the case that the simplification necessary to express emissions per functional unit in a static model leads to rather restrictive assumptions and a degree of reduction that causes this sensitivity. It should be made clear that the merits of using a clear functional unit are not challenged here, but the method of comparing outcomes of a study might actually lead to a reduction that is inappropriate; the method of expressing emissions per functional unit and using these as a basis of comparison in a policy context, instead of using the total system output (i.e., total volumes of emissions and functions over time) given the system boundary. Other authors [35] have also reflected on this issue from a supply chain management perspective. The problem here is not only one of aggregation-which cannot be avoided in many cases-but also of time dependency (e.g., how emissions are weighted throughout time).

Secondly, in the linear and static approach, strong assumptions need to be made about the recyclability of products in order to be able to perform allocation procedures when there are recycling loops. For instance, in order to use some known standard equations for closed 
or open loop recycling systems, the modeler must, amongst others, estimate how often a product will be recycled. Such a parameter is typically modelled as an exogenous and constant fraction, whereas in reality it is more likely that this fraction is in fact endogenous, i.e., there is heterogeneity and a distribution of how often a certain product can be recycled with different pathways, depending on technological change, human behavior, and the availability of waste management systems. Moreover, there may be significant accumulation effects taking place that cannot be captured by a model that assumes equilibrium, and could have a profound impact on model outcomes (e.g., nonlinear capacity constraints). Again, when the study somehow aims to support decision-making at the policy level of a company, NGO, government, or other stakeholder, model robustness is sensitive to assumptions regarding its boundary, not just its data availability. Perhaps, in that respect, more attention should be given towards system expansion methods, especially in cLCA, to avoid skewed results.

\subsubsection{Dynamic LCA}

In the early years of this century, the concept of DLCA emerged. This was done in response to the methodological limitations of traditional LCA methods which, amongst others, do not consider factors that exhibit time varying behavior. Ever since, various authors have contributed to the methodological discussion surrounding LCA and DLCA, as well as numerous examples of applications of DLCA.

A recent $\mathrm{PhD}$ thesis [36] pointed out that it is essential to model temporal characteristics in order to capture the transient behavior of complex processes and supply chains. This is also due to the fact that the impacts of anthropogenic emissions are time dependent due to absorptive capacities which depend on the concentration profiles temporally and spatially. To introduce dynamism, the author created a tool to import the technology matrix $A$ (see Equation (1)) and include time dependency, which can be found in other works as well $[37,38]$.

Since the inventory step in the LCA describes production systems and such systems are dynamic, it is likely that their dynamics are influential in the outcome of LCA studies [27,36]. Other authors [39] argue that especially for products with long life cycles, such as, in their case, buildings, their performance might be affected significantly by economic, environmental and societal changes. The timing of emissions matters in such cases. This can be seen in another study [40], where the author performed a DLCA on renewable energy and suggests that future developments and context is typically not considered in regular LCA approaches, thereby "severely distorting the analysis of the environmental characteristics of future energy systems". This does not only play a role in production systems or energy markets but has been observed by other authors as well in agricultural systems, where dynamic factors can have a strong influence on the outcome of LCA studies [41,42].

Other authors [37] performed a sensitivity analysis of temporal parameters in a DLCA framework and showed that it is important to study the emissions' profile during the lifetime of the system, as well as several decades afterwards in order to understand accumulation effects.

In a recent review paper on DLCA [7], which aimed to assess the state of the art in DLCA applications and the various types of DLCA that were involved, the authors distinguished between three types of LCA dynamisms:

1. dynamic process inventory: Potential future developments are accounted for in unit-processes;

2. dynamic systems inventory: Potential future changes in components of the system under study are modelled by switching between unit processes or behaviors;

3. dynamic characterization: adjusting factors to account for temporality in emissions of for example, $\mathrm{CO}_{2}$ equivalency (future versus present day).

The vast majority (93\%) of the papers they analyzed did not develop and use a fully dynamic model, but only partially accounted for dynamism. They proceeded to assert 
that there are ample cases where the use of a partially dynamic LCA would provide better decision support than the conventional static LCA, but at the same time also caution that standards should be set as to how one should implement and report on (partially) dynamic LCAs to improve consistency and avoid potentially skewed results, which could result from arbitrary choices and assumptions in dynamism.

Indeed, it seems the literature regarding DLCA is still in its infancy, considering that very few articles include fully dynamic models. As outlined above, the timing of emissions and the efficiency and response of background systems play an important role. Here, background systems are defined as being part of the supply chain, but outside the sphere of influence of the decision-makers [43]. A prime example of how the timing of emissions and the background system can strongly determine the sustainability of a product can be found in [44], where the burning of wood pellets is shown to be much worse in terms of greenhouse gas (GHG) emissions than using coal fired power in the short-term. The goal of their study was to assess to what degree burning wood lowers $\mathrm{CO}_{2}$ emissions compared to coal, and emphasized that such a question requires dynamic analysis. Figure 2 provides a snapshot of their findings and particularly the time it takes to pay back the initial carbon debt that is incurred when burning wood pellets. The scenarios 1 through 5 consider the following:

(a) Scenario 1: Burning wood pellets has the same combustion, processing, and supply chain efficiency as coal. Interestingly, $25 \%$ of biomass harvested from the land occurred through thinning;

(b) Scenario 2: Same as Scenario 1 but now with the actual efficiencies and supply chain emissions for wood;

(c) Scenario 3: Scenario 2 but with 95\% of biomass harvested (clear cut);

(d) Scenario 4: Clear cut, no regrowth of harvested wood, and no carbon emissions from soil stocks;

(e) Scenario 5: Scenario 4 but with carbon emissions from soil stocks.

Even in highly unrealistic scenarios (S1 through S3) that are favorable for the biomass configuration, it can take up to 80 years to pay back the carbon debt. These scenarios assume optimistic wood cutting and forest management practices, no ecological disturbances such as fires, erosion, disease, and supply chain emissions of wood equal to that of coal per end-use energy. Scenarios that are not artificially favorable ( $\mathrm{S} 4$ and beyond) show that burning wood in fact increases $\mathrm{CO}_{2}$ emissions relative to coal.

Remarkably, another set of authors who published their LCA study around the same time found that it only takes a few years to pay back the carbon debt under various assumptions regarding conversion efficiency, biomass decay half-life period, and emission factors (Figure 3). Even though there are some differences in estimating supply chain emissions, both articles agree that up until the point of combustion, burning wood pellets emits more $\mathrm{CO}_{2}$ than coal. Yet their outcomes suggest different conclusions concerning the sustainability of burning wood pellets.

The differences exist mainly due to the fact that [45] (Figure 3), while including a dynamic component in their study, is the only $\mathrm{CO}_{2}$ emissions model that pertains to the decay of biomass in the counterfactual scenario-i.e., the wood pellets cause emissions due to decay if they are not combusted. Moreover, [44], in addition to modelling decay, included a more detailed and dynamic background of forest growth, capturing non-linearities and feedback effects in the carbon cycle of biomass which depend on more factors than are typically covered by conventional LCA methods. One of the reasons for these differences in modelling is that in the LCA of [45], the wood pellets used in combustion are considered to be sourced from wood waste and forest residues instead of dedicated harvest, and are thus generated independently of bioenergy demand. Still, explicit modelling of the background system (as was the case in [44]) is a more comprehensive and consistent way of yielding an integrated assessment under various assumptions. 


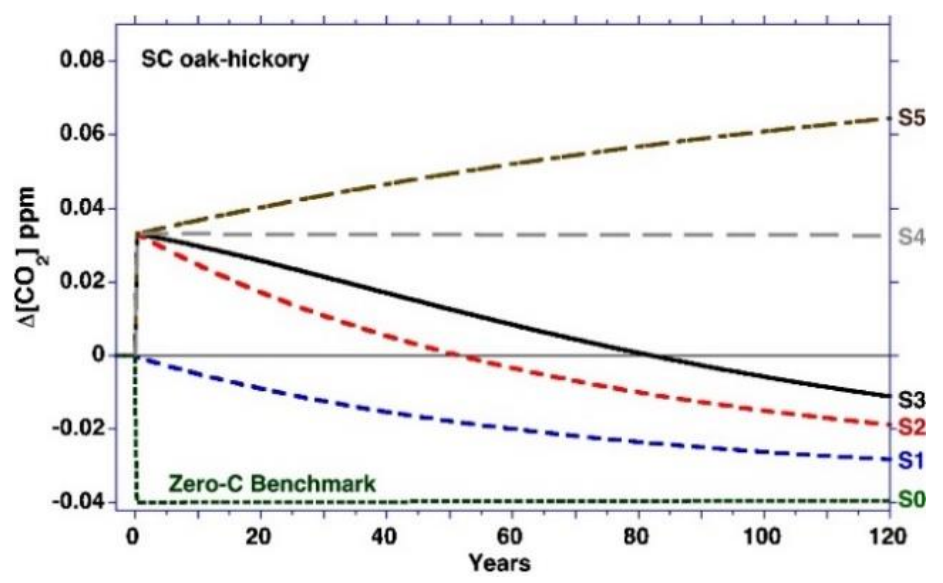

(a)

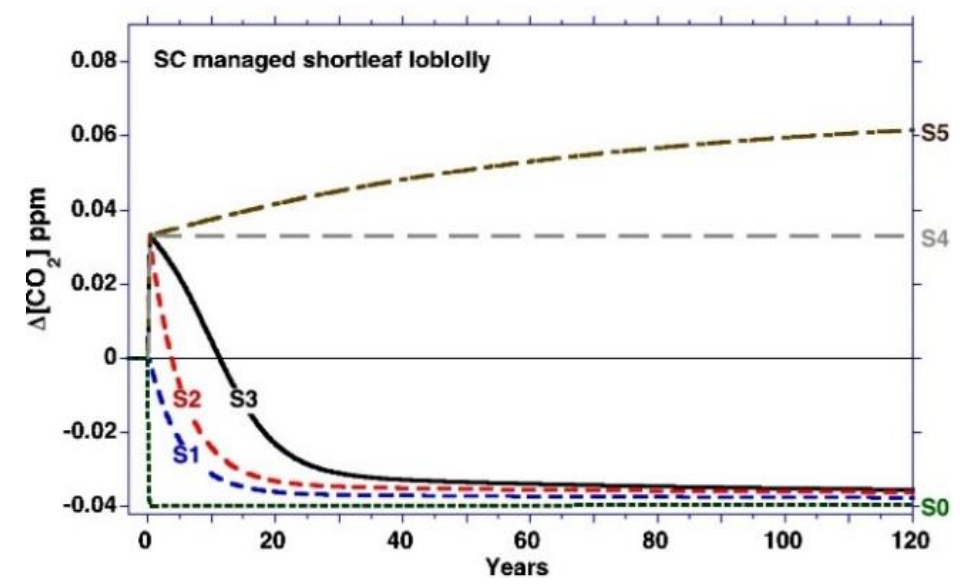

(b)

Figure 2. The effects of displacing coal with wood on changes in atmospheric $\mathrm{CO}_{2}$ concentration. (a) Displacing coal with wood cut from south-central oak-hickory forest; (b) displacing coal with wood cut from south-central managed shortleaf loblolly. Figure taken from [44].

This specific comparison serves as an example to show that the emissions profile and dynamics of background systems (in this case forest growth and forest management) play an important role in impact assessment, and are typically excluded in conventional (static) LCAs. In addition, the lessons learned from this example may also be relevant for biopolymer system analyses, as they too rely on the premise of fossil fuel substitution through feedstock obtained from biomass.

In other work, [46] applied both dynamic process inventory and dynamic characterization in their study. They developed characterization factors for global warming dynamically and refuted the claim that corn-based ethanol has a better GHG impact than gasoline, which they based on a static LCA. Another study found that, for $8.6 \%$ of the database of product systems in LCI data, global warming impact scores were affected by more than $10 \%$ if temporal effects were modelled in the background system [47].

\subsubsection{Ex-ante LCA and Multi Method Approaches}

As interest in LCA related methods and applications has risen throughout the last decades, so have the number of publications and methodological advances in the field. Recently, [48] discussed an application referred to as 'ex-ante' LCA. Rather than performing an 'ex-post' LCA, the 'ex-ante' approach helps assess the projected future of emerging technologies. This can be an instrumental tool in aiding technology design and development, as early decisions in the design process often have far reaching consequences in the future. Therefore, early decision support through ex-ante LCA can be instrumental 
in minimizing important footprints. The authors define ex-ante LCA as being studies that compare scaled-up emerging technologies with evolved incumbent technologies and scaled-up emerging technologies based on likely scenarios in terms of their future performance when operational. In the context of bio-based chemicals, [49] found that when starting at the process design phase of a new production process, ex-ante LCA yielded good predictions of hotspots. This allows for an early identification of major contributions to the emissions pertaining to a product's life cycle.
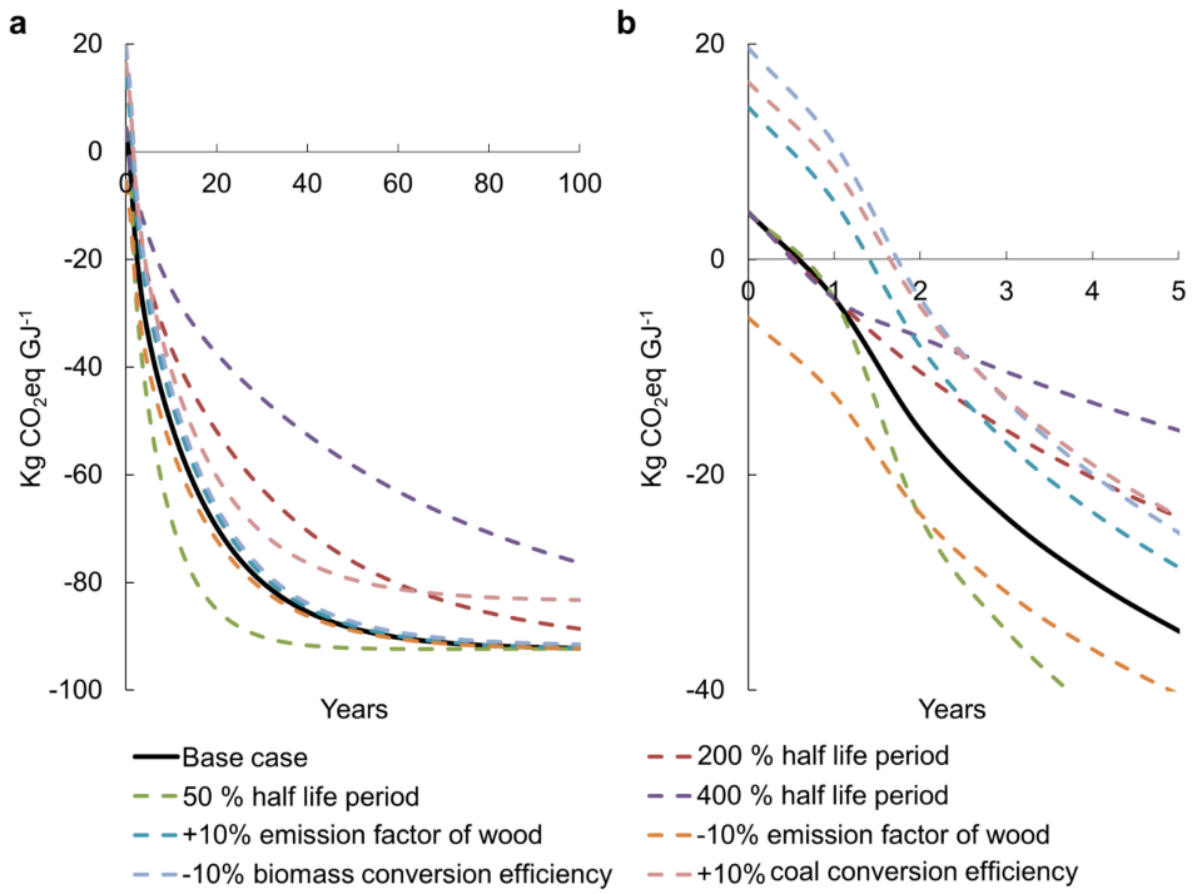

Figure 3. Greenhouse gas (GHG) emissions of wood displacing coal. (a) The net $\mathrm{CO}_{2}$ savings per end-use energy under varying assumptions of key parameters; (b) equal to (a) but zoomed in. Figure taken from [45].

In another paper, [50] reviewed case studies pertaining to ex-ante LCA and proposed a framework wherein the emerging technology should be compared with an incumbent technology and interact with the background system at three different moments in time: the current situation, the time at which the emerging technology reaches maturity, and, finally, a point in time where the mature technology was implemented for a while already. In terms of categorizing modelled aspects in their reviewed articles, they used the categories 'Technology Development', 'Technological Learning', and 'Technology Diffusion'. The majority of studies in their review sample only partially modelled at most two of these aspects, while all are relevant in assessing the prospects of emerging technologies.

Due to its forward looking nature and evaluation of an emerging technology, ex-ante LCA facilitates the practice of combining LCA with other methods such as technoeconomic analysis (TEA), life cycle costing (LCC), social life cycle assessment (sLCA), multi criteria decision analysis (MCDA), and others. Recently, [51] wrote a review on LCA of emerging technologies and discussed the use of such and other methods in conjunction with LCA, as well as the challenges that exist in performing LCA on emerging technologies at early development stages. They identified an opportunity in further integration of LCA with TEA. In other work, [24] systematically reviewed papers on prospective LCA for emerging technologies. Even though they did not refer to these LCA studies as 'ex-ante', many of them fit this terminology considering their scope and aim. They found that there was a marked increase in such applications in recent years, but point out that there exists a lot of uncertainty regarding transparency and justification of data quality in the applications they reviewed. The same goes for system boundaries and how uncertainty was handled. 
They call for a more standardized approach in order to improve comparability between such studies.

In other LCA studies where the aim is not necessarily to perform an ex-ante study, there are ample opportunities to apply other methods to support or extend the analysis. For instance, MCDA, TEA, and life cycle costing (LCC) are just a few examples of methods that can be found in some recent applications, although these are not yet widely used. This also includes the application of linear programming methods in consequential approaches, as can be found in [52]. A recent stream of LCA called the life cycle sustainability Assessment (LCSA) tends to embrace such a multi method approach [53,54]. Several years ago, [55] performed a review on LCSA applications and found that methods to deepen LCSA were lacking, proposing systems thinking and related tools as potentially valuable to deepen the analyses.

\section{An Overview of Recent Plastics and Recycling LCA Studies}

A literature search was conducted in WorldCat to find peer-reviewed studies in the past five years (2016-2020) that performed an LCA on plastic (products) and which explicitly cover end-of-life (EoL) and recycling to assess the current state-of-the-art information. Figure 4 shows the search criteria and selection criteria for deriving the final list of papers tabulated in Table 2.

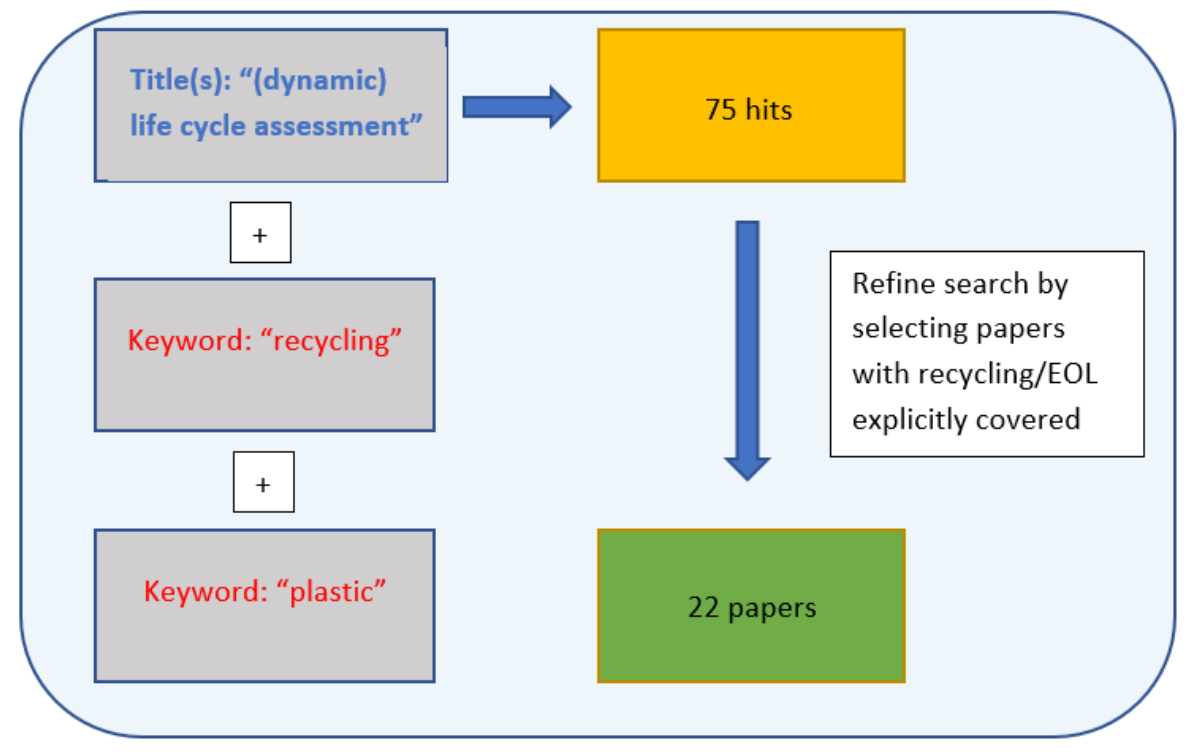

Figure 4. Selection criteria for reviewed papers.

The initial search yielded 75 hits, which were further refined after screening by selecting only those papers with an LCA application relevant to recycling/end-of-life in the context of plastics, resulting in 22 articles. To ensure we included applications that pursued dynamic analysis, the title term was changed to 'dynamic life cycle assessment', which yielded 7 additional results. While some of those articles are discussed in other sections of this paper, none of them fell within the scope of plastics and recycling, so they have been omitted from the comparison in this section. Moreover, this overview is not meant to be exhaustive, but rather a snapshot of recent contributions that give insight in the methodological considerations found in recent years. The papers are tabulated according to the type of LCA, the choice of functional unit, system boundaries, dynamism, accounting method (based on Table 1), interaction effects (feedback) with background systems, multi-method, and how model uncertainty is accounted for. 


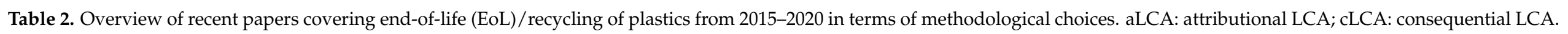

\begin{tabular}{|c|c|c|c|c|c|c|c|c|}
\hline Authors & LCA Type & Functional Unit & System Boundaries & Dynamism & Accounting Method & Feedback Effects & Multi-Method & Model Uncertainty \\
\hline [56] & aLCA & Mass & Gate-to-grave/Gate-to-cradle & No & A form of partitioning * & No & No & No \\
\hline [57] & aLCA & Volume + Time & Cradle-to-cradle & No & $\mathrm{N} / \mathrm{A} *$ & No & No & Sensitivity Analysis \\
\hline [58] & aLCA & Area & Gate-to-grave & No & Cut-off & No & No & No \\
\hline [59] & aLCA & Mass & Cradle-to-grave & No & $\mathrm{N} / \mathrm{A} *$ & No & Yes & No \\
\hline [60] & aLCA & Mass & Gate-to-cradle & No & Cut-off & No & No & Sensitivity Analysis \\
\hline [61] & aLCA & Mass & Gate-to-Gate & No & Partitioning & No & No & No \\
\hline [62] & cLCA & Mass & Cradle-to-Gate & No & End-of-Life Recycling & No & Yes & Sensitivity Analysis \\
\hline [63] & Ex-ante LCA & Mass & Cradle-to-Gate & No & System Expansion & No & No & No \\
\hline [64] & CLCA & Mass + Distance & Gate-to-Grave/Cradle & No & End-of-Life Recycling & No & No & Sensitivity Analysis \\
\hline [65] & cLCA & Mass & Gate-to-Grave/Cradle & No & System Expansion & No & No & No \\
\hline [66] & aLCA & Mass & Gate-to-Cradle & No & Partitioning & No & No & Sensitivity Analysis \\
\hline [67] & $\mathrm{aLCA}^{*}$ & Volume/Mass & Gate-to-Grave/Cradle & No & $\begin{array}{l}\text { Cut-off and System Expansion } \\
\text { (they call it substitution) }\end{array}$ & No & No & No \\
\hline [68] & cLCA & Mass & Gate-to-Cradle & No & System Expansion & No & Yes & Sensitivity Analysis \\
\hline [69] & aLCA & Area & Cradle-to-Grave/Cradle & No & $\mathrm{N} / \mathrm{A} *$ & No & Yes & No \\
\hline [70] & aLCA & Mass & Gate-to-Cradle & No & Cut-off and System Expansion & No & No & No \\
\hline [71] & CLCA & Mass & Gate-to-Cradle & No & End-of-Life Recycling * & No & $\mathrm{No}^{*}$ & Sensitivity Analysis \\
\hline [72] & cLCA & Mass/Volume & Cradle-to-Grave/Cradle & No & End-of-Life Recycling * & No & No & Sensitivity Analysis \\
\hline [73] & cLCA & Mass & Gate-to-Cradle/Grave & No & System Expansion & No & No & Sensitivity Analysis \\
\hline$[74]$ & CLCA & Mass & Gate-to-Gate & No & System Expansion & No & Yes & No \\
\hline [75] & CLCA & Mass & Cradle-to-Gate* & No & $\mathrm{N} / \mathrm{A}^{*}$ & No & No & Monte Carlo + Sensitivity Analysis \\
\hline
\end{tabular}

* denotes methodological choices that were inferred from their original source. 


\section{Results}

Half of the investigated studies used an attributional approach. A further 10 out of 22 used a consequential approach, while only one study performed an ex-ante LCA. More than $80 \%$ of the papers used mass as a functional unit. As can be seen in Table 2, a variety of accounting methods were used, with system expansion and EoL recycling being the most popular. There is one side note, however-in approximately $30 \%$ of the studies it was not fully clear what method was used as the authors were not explicit about their methodological choice in that respect. These studies have been denoted with an asterisk in Table 2.

No dynamism or interaction effects (feedback) between background and foreground systems were modelled in these sample papers. A total of five papers combined at least one other method with LCA to enrich the outcome of their study. While it was clear that high standards of data quality were met in these studies, it is rather unfortunate that parametric uncertainty was only covered in half the contributions by means of sensitivity analysis, and only in two cases were more robust and rigorous routines such as Monte Carlo simulation considered. It has been found before that only applying sensitivity analysis can yield misleading results [34].

\section{Discussion}

The current state of research seems to be in line with other studies on LCA practices, as found, for example, in a review paper on LCA applications in the chemical industry [78]. In addition, no explicit additional methods were covered to provide the means necessary for policy analysis, even though, in a larger context, it is clear that there are decision-makers who need to decide upon waste management strategies that may or may not include certain recycling methods. In essence, even though consequential approaches in particular are seen as appealing tools for policy-makers [22,79], there still exists a gap between the modelled system, which is a strong reduction of the real system, and the context of decision-making. The gap between LCA and policy is also recognized by [33]. If LCA is to be used as decision support for policy-makers, it should model interactions between relevant fore and background systems, and not simply assume a static world.

\section{Bridging the Gap?}

As was outlined in the previous sections, a combination of methods is seen as a promising new route for LCA studies to enrich their outcomes. Considering the importance of the plastics industry, it would be an interesting and potentially important line of research to set up LCA studies that actively seek to integrate multiple methods so as to enhance the decision-making process at all relevant levels in industry and government. However, despite the advent of new streams of LCA such as LCSA and ex-ante approaches, there still exists a void that needs to be filled in order to better suit the needs of decision-makers. This would especially be the case for more prospective oriented studies, as decisions in the near future affect the real world system for many years to come. In such situations, it is desirable to be able to project the evolution of a product system throughout time. Yet dynamism itself is most likely not enough. To improve the models so that they can become a better support for decision-making, it is also important that such models include behavioral feedbacks where necessary, to capture interactions that exist between, for example, consumer behavior and EoL processes, thereby being able to assess the potential impacts of consumer behavior on emissions in the EoL phase(s).

As a prime example, consider the current worldwide crisis in plastics pollution, specifically in the oceans. The availability of waste management systems and human behavior are prime determinants of these environmental flows $[3,80]$. In a hypothetical case, suppose decision-makers can select from three competing products or recycling processes, which have different qualities (e.g., durability, disposability, and recyclability). Besides differences in material and energy flows in these product systems resulting from their functionality, the product qualities would likely affect the behavior of agents (for 
instance consumers) within the supply chain. From a policy perspective, such effects matter. Yet conventional LCA does not offer any guidelines on how to include such effects and therefore omits these processes.

No matter how accurate one's data is on any supply chain for a particular product configuration, or how efficient a new recycling process is, none of these really matter if the real world system is subject to completely different behavior than assumed in any of our models. While modelling human behavior or socioeconomic conditions might seem hard or impossible, the exclusion of such variables is hard to justify when they are known to be central to a problem. Basing decisions on strongly reduced models which are linear, steady state, and exclude important feedbacks known to exist in the real world would be uninformed at best, which is demonstrated by the biomass case discussed earlier.

To this end, there are other fields that might be able to deliver important contributions to the well-recognized LCA community. In particular, dynamic modelers active in disciplines such as operations research (OR) might be able to help transform the static approaches to dynamic ones through the use of discrete event simulation (DES), system dynamics (SD), or agent-based modelling (ABM). These techniques have proven to be effective in capturing behavioral components in value chains and creating models suitable for policy analysis [81]. So far, to the best of the authors' knowledge, there has been little cross fertilization between LCA and such modelling methods. According to [55], the first study that integrated SD into LCSA analyzed the sustainability of different vehicle technologies [82]. Recently, [83] reported that they were able to successfully recreate an LCA study in SD software, highlighting the integration of both methods as they reinforced each other. Furthermore, [44] showed how dynamic analysis can answer broader research questions (e.g., "Is X better than Y to improve Z?"), of particular importance at the level of policy- and decision-makers. Another relatively recent paper by [84] used agent-based modelling to assess possible emergent behavior at the LCI stage of an LCSA, thereby showing it is feasible to incorporate ABM into the LC(S)A framework. Finally, [35] provided an interesting characterization of LCA and how modelling principles from supply chain management can contribute to the practice of LCA, by emphasizing the complex, nonlinear character of supply chains, focusing on robustness of decisions rather than confidence intervals for impacts, and how incentives matter, amongst others. They too recognize the importance of focusing on the performance of the (product system) as a whole, rather than per unit, as argued before.

Still, even though the inclusion of dynamic interactions and behavioral processes would allow modelers to enrich their analyses, it does not solve the complexity of setting system boundaries and related assumptions. There is also a need to be able to assess the relative sensitivity of a model to its chosen boundaries, not just the parametric uncertainty, as it is precisely the relatively narrow model boundaries of LCAs that hamper its ability to accurately estimate real world impacts when evaluating policy options. As was seen in Table 1, approximately half of the papers used sensitivity analysis to investigate the possible behavior space of their models. Of course, Monte Carlo simulation and related routines can further enhance these analyses, but this does not fundamentally address any uncertainty that extends beyond parametric uncertainty.

Sometimes there is no prior knowledge on input or output distributions of certain processes or variables or interconnections in the system, especially once the model boundary is extended beyond the inclusion of only (bio)-physical flows. In addition, there might be disagreement between modelers and stakeholders on what should be included in the model boundary or how to model this appropriately. It is not unlikely that ex-ante or consequential studies in particular face such problems, especially during the process of expanding model boundaries. In these cases, dealing with what is known as deep uncertainty, more advanced (sampling) methods can be an outcome as proposed by [85]. Such methods also sample over the uncertainties that exist when adopting one or multiple policies and how these interact with each other and the system under study, thereby exploring the policy space. Here, policy refers to decisions. Thus, evaluating the effects of different 
system configurations in a cLCA setting could be seen as testing 'policies', for example. The techniques proposed in that paper allow modelers to perform scenario discovery analyses and robust decision-making, in line with the proposed value of supply chain management principles outlined by [35] (e.g., robustness of decisions). Moreover, the use of such methods for deep uncertainty also addresses one of the core issues raised by [86] concerning the management of uncertainty in LCA.

In summary, three future research lines could be beneficial for LCA practitioners and researchers active in modelling product systems for the plastics and recycling industry:

1. Mix methods by including dynamic modelling practices such as for example DES, SD, and/or ABM to allow for the inclusion of temporal and spatial dynamics;

2. Use the aforementioned or other methods to model the dynamics between fore and background systems and the wider context in order to facilitate policy analysis;

3. Use more rigorous sampling methods to investigate model behavior and consider using tools and techniques pertaining to deep uncertainty to assess policy options for their robustness, so that potential real world effects can be investigated and policymakers can be supported in long term planning.

\section{Conclusions}

The majority of recent studies concerning plastics and recycling are still relatively conservative in how LCAs are performed, while the methodological diversity in LCA has expanded over the last decade. Future studies should consolidate new methodological advances and consider integrating dynamic modelling practices and advanced sampling methods to further strengthen the quality of LCA models as a support for decision-making, as decision-makers have to make design or regulatory choices at a system level that is higher and broader than what is typically modelled in an LCA. This is particularly for consequential and ex-ante studies, as recent dynamic analyses have shown that a steady-state approach can draw erroneous and misguided policy conclusions. In addition, dynamism itself is not enough: LCA model boundaries must be broadened to provide better support for policy making and early design choices in the life cycle of a new product or system.

Author Contributions: Conceptualization, T.M.K.; methodology, T.M.K.; resources, A.I.V.; writingoriginal draft preparation, T.M.K., G.H.J.; writing—review and editing, T.M.K., G.H.J., and A.I.V.; supervision, A.I.V. All authors have read and agreed to the published version of the manuscript.

Funding: This research received no external funding.

Institutional Review Board Statement: Not applicable.

Informed Consent Statement: Not applicable.

Data Availability Statement: Not applicable.

Acknowledgments: The authors are grateful to F. Picchioni for useful discussions on the topics of this paper.

Conflicts of Interest: The authors declare no conflict of interest.

\section{References}

1. Dhanumalayan, E.; Joshi, G. Performance properties and applications of polytetrafluoroethylene (PTFE)—A review. Adv. Compos. Hybrid Mater. 2018, 1. [CrossRef]

2. McKeen, L.W. 6-Polyester Plastics. In Permeability Properties of Plastics and Elastomers, 4th Ed.; McKeen, L.W., Ed.; Plastics Design Library; William Andrew Publishing: Norwich, NY, USA, 2017; pp. 95-114. [CrossRef]

3. McKinsey Center. Ocean Conservancy. Stemming the Tide: Land-Based Strategies for a Plastic-Free Ocean; Ocean Conservancy: Washington, DC, USA; McKinsey Center for Business and Environment: Chicago, IL, USA, 2015; p. 47.

4. Sala, S.; Reale, F.; Cristóbal-García, J.; Marelli, L.; Rana, P. Life Cycle Assessment for the Impact Assessment of Policies. Life Thinking and Assessment in the European Policies and for Evaluating Policy Options; European Commission: Ispra, Italy, 2016; Volume 28380. [CrossRef]

5. Kloepffer, W. Life cycle sustainability assessment of products. Int. J. Life Cycle Assess. 2008, 13, 89. [CrossRef] 
6. Guinée, J.B.; Heijungs, R.; Huppes, G.; Zamagni, A.; Masoni, P.; Buonamici, R.; Ekvall, T.; Rydberg, T. Life Cycle Assessment: Past, Present, and Future. Environ. Sci. Technol. 2011, 45, 90-96. [CrossRef] [PubMed]

7. Sohn, J.; Kalbar, P.; Goldstein, B.; Birkved, M. Defining Temporally Dynamic Life Cycle Assessment: A Review. Integr. Environ. Assess. Manag. 2020, 16, 314-323. [CrossRef] [PubMed]

8. Hunt, R.G.; Franklin, W.E. LCA-How it Came About. Int. J. LCA 1996, 1, 4-7. [CrossRef]

9. Klöpffer, W. The role of SETAC in the development of LCA. Int. J. Life Cycle Assess. 2006, 11, 116-122. [CrossRef]

10. ISO14040. Environmental management_Life Cycle Assessment_Part 1: Principles and Framework; ISO: Geneva, Switzerland, 1997.

11. ISO. ISO/IEC 14044:2006 Environmental Management_Life Cycle Assessment_Requirements and Guidelines; ISO: Geneva, Switzerland, 2006.

12. Klopffer, W.; Grahl, B. Life Cycle Assessment (LCA): A Guide to Best Practice; Wiley-VCH: Weinheim, Germany, 2014.

13. Rebitzer, G.; Ekvall, T.; Frischknecht, R.; Hunkeler, D.; Norris, G.; Rydberg, T.; Schmidt, W.P.; Suh, S.; Weidema, B.P.; Pennington, D.W. Life cycle assessment Part 1: Framework, goal and scope definition, inventory analysis, and applications. Environ. Int. 2004, 30, 701-720. [CrossRef]

14. Guinée, J.B. Selection of Impact Categories and Classification of LCI Results to Impact Categories. In Life Cycle Impact Assessment; Springer Science \& Business Media: Dordrecht, The Netherlands, 2015; pp. 17-37.

15. Heijungs, R.; Suh, S. The Computational Structure of Life Cycle Assessment; Kluwer: Dordrecht, The Netherlands, 2002.

16. Brander, M. Conceptualising attributional LCA is necessary for resolving methodological issues such as the appropriate form of land use baseline. Int. J. Life Cycle Assess. 2016, 21, 1816-1821. [CrossRef]

17. Sonnemann, G.; Vigon, B. Global Guidance Principles for Life Cycle Assessment Databases: A Basis for Greener Processes and Products; UNEP: Paris, France, 2011.

18. Soimakallio, S.; Cowie, A.; Brandão, M.; Finnveden, G.; Ekvall, T.; Erlandsson, M.; Koponen, K.; Karlsson, P.E. Attributional life cycle assessment: Is a land-use baseline necessary? Int. J. Life Cycle Assess. 2015, 20, 1364-1375. [CrossRef]

19. Curran, M.A.; Mann, M.; Norris, G. The international workshop on electricity data for life cycle inventories. J. Clean. Prod. 2005, 13, 853-862. [CrossRef]

20. Ekvall, T.; Weidema, B.P. System boundaries and input data in consequential life cycle inventory analysis. Int. J. Life Cycle Assess. 2004, 9, 161-171. [CrossRef]

21. Martin, E.W.; Chester, M.V.; Vergara, S.E. Attributional and Consequential Life-cycle Assessment in Biofuels: A Review of Recent Literature in the Context of System Boundaries. Curr. Sustain. Energy Rep. 2015, 2, 82-89. [CrossRef]

22. McManus, M.C.; Taylor, C.M. The changing nature of life cycle assessment. Biomass Bioenergy 2015, 82, 13-26. [CrossRef] [PubMed]

23. Ekvall, T.; Tillman, A.M.; Molander, S. Normative ethics and methodology for life cycle assessment. J. Clean. Prod. 2005, 13, 1225-1234. [CrossRef]

24. Thonemann, N.; Schulte, A.; Maga, D. How to conduct prospective life cycle assessment for emerging technologies? A systematic review and methodological guidance. Sustainability 2020, 12, 1192. [CrossRef]

25. ISO 14041 Environmental management_Life Cycle Assessment_Goal and Scope Definition and Inventory Analysis; ISO: Geneva, Switzerland, 1998.

26. Jones, C.; Gilbert, P.; Raugei, M.; Mander, S.; Leccisi, E. An approach to prospective consequential life cycle assessment and net energy analysis of distributed electricity generation. Energy Policy 2017, 100, 350-358. [CrossRef]

27. Reap, J.; Roman, F.; Duncan, S.; Bras, B. A survey of unresolved problems in life cycle assessment. Part 1: Goal and scope and inventory analysis. Int. J. Life Cycle Assess. 2008, 13, 290-300. [CrossRef]

28. Schrijvers, D.L.; Loubet, P.; Sonnemann, G. Developing a systematic framework for consistent allocation in LCA. Int. J. Life Cycle Assess. 2016, 21, 976-993. [CrossRef]

29. Heijungs, R. Ten easy lessons for good communication of LCA. Int. J. Life Cycle Assess. 2014, 19, 473-476. [CrossRef]

30. Ekvall, T.; Tillman, A.M. Open-loop recycling: Criteria for allocation procedures. Int. J. Life Cycle Assess. 1997, 2, 155-162. [CrossRef]

31. Frischknecht, R. LCI modelling approaches applied on recycling of materials in view of environmental sustainability, risk perception and eco-efficiency. Int. J. Life Cycle Assess. 2010, 15, 666-671. [CrossRef]

32. European Union. Commission Recommendation of 9 April 2013 on the Use of Common Methods to Measure and Communicate the Life Cycle Environmental Performance of Products and Organisations; European Union: Brussels, Belgium, 2013; Volume 56.

33. Wardenaar, T.; Van Ruijven, T.; Beltran, A.M.; Vad, K.; Guinée, J.; Heijungs, R. Differences between LCA for analysis and LCA for policy: A case study on the consequences of allocation choices in bio-energy policies. Int. J. Life Cycle Assess. 2012, 17, 1059-1067. [CrossRef]

34. Cherubini, E.; Franco, D.; Zanghelini, G.M.; Soares, S.R. Uncertainty in LCA case study due to allocation approaches and life cycle impact assessment methods. Int. J. Life Cycle Assess. 2018, 23, 2055-2070. [CrossRef]

35. Blass, V.; Corbett, C.J. Same Supply Chain, Different Models: Integrating Perspectives from Life Cycle Assessment and Supply Chain Management. J. Ind. Ecol. 2018, 22, 18-30. [CrossRef]

36. Shimako, A. Contribution to the Development of a Dynamic Life Cycle Assessment Method. Ph.D. Thesis, INSA de Toulouse, Toulouse, France, November 2017. 
37. Shimako, A.H.; Tiruta-Barna, L.; Bisinella de Faria, A.B.; Ahmadi, A.; Spérandio, M. Sensitivity analysis of temporal parameters in a dynamic LCA framework. Sci. Total Environ. 2018, 624, 1250-1262. [CrossRef] [PubMed]

38. Pigné, Y.; Gutiérrez, T.N.; Gibon, T.; Schaubroeck, T.; Popovici, E.; Shimako, A.H.; Benetto, E.; Tiruta-Barna, L. A tool to operationalize dynamic LCA, including time differentiation on the complete background database. Int. J. Life Cycle Assess. 2020, 25, 267-279. [CrossRef]

39. Su, S.; Li, X.; Zhu, Y.; Lin, B. Dynamic LCA framework for environmental impact assessment of buildings. Energy Build. 2017, 149, 310-320. [CrossRef]

40. Pehnt, M. Dynamic life cycle assessment (LCA) of renewable energy technologies. Renew. Energy 2006, 31, 55-71. [CrossRef]

41. Aktar, W.; Sengupta, D.; Chowdhury, A. Impact of pesticides use in agriculture: Their benefits and hazards. Interdiscip. Toxicol. 2009, 2, 1-12. [CrossRef]

42. Lan, K.; Yao, Y. Integrating Life Cycle Assessment and Agent-Based Modeling: A Dynamic Modeling Framework for Sustainable Agricultural Systems. J. Clean. Prod. 2019, 238, 117853. [CrossRef]

43. Tillman, A.M. Significance of decision-making for LCA methodology. Environ. Impact Assess. Rev. 2000, 20, 113-123. [CrossRef]

44. Sterman, J.D.; Siegel, L.; Rooney-Varga, J.N. Does replacing coal with wood lower $\mathrm{CO}_{2}$ emissions? Dynamic lifecycle analysis of wood bioenergy. Environ. Res. Lett. 2018, 13, 015007. [CrossRef]

45. Madsen, K.; Bentsen, N.S. Carbon debt payback time for a biomass fired CHP plant-A case study from northern Europe. Energies 2018, 11, 807. [CrossRef]

46. Levasseur, A.; Lesage, P.; Margni, M.; Deschěnes, L.; Samson, R. Considering time in LCA: Dynamic LCA and its application to global warming impact assessments. Environ. Sci. Technol. 2010, 44, 3169-3174. [CrossRef]

47. Pinsonnault, A.; Lesage, P.; Levasseur, A.; Samson, R. Temporal differentiation of background systems in LCA: Relevance of adding temporal information in LCI databases. Int. J. Life Cycle Assess. 2014, 19, 1843-1853. [CrossRef]

48. Cucurachi, S.; Van Der Giesen, C.; Guinée, J. Ex-ante LCA of Emerging Technologies. Procedia CIRP 2018, 69, 463-468. [CrossRef]

49. Fernandez-Dacosta, C.; Wassenaar, P.N.H.; Dencic, I.; Zijp, M.C.; Morao, A.; Heugens, E.H.W.; Shen, L. Can we assess innovative bio-based chemicals in their early development stage? A comparison between early-stage and life cycle assessments. J. Clean. Prod. 2019, 230, 137-149. [CrossRef]

50. Buyle, M.; Audenaert, A.; Billen, P.; Boonen, K.; Van Passel, S. The future of ex-ante LCA? Lessons learned and practical recommendations. Sustainability 2019, 11, 5456. [CrossRef]

51. Moni, S.M.; Mahmud, R.; High, K.; Carbajales-Dale, M. Life cycle assessment of emerging technologies: A review. J. Ind. Ecol. 2020, 24, 52-63. [CrossRef]

52. Budzinski, M.; Sisca, M.; Thrän, D. Consequential LCA and LCC using linear programming: An illustrative example of biorefineries. Int. J. Life Cycle Assess. 2019, 24, 2191-2205. [CrossRef]

53. Guinée, J. Life cycle sustainability assessment: What is it and what are its challenges? In Taking Stock of Industrial Ecology; Springer: Cham, Switzerland, 2015; pp. 45-68. [CrossRef]

54. Kucukvar, M. Life Cycle Sustainability Assessment Framework for the U.S. Built Environment. Ph.D. Thesis, University of Central Florida, Orlando, FL, USA, December 2014.

55. Onat, N.C.; Kucukvar, M.; Halog, A.; Cloutier, S. Systems thinking for life cycle sustainability assessment: A review of recent developments, applications, and future perspectives. Sustainability 2017, 9, 706. [CrossRef]

56. Nunes, A.O.; Viana, L.R.; Guineheuc, P.M.; da Silva Moris, V.A.; de Paiva, J.M.F.; Barna, R.; Soudais, Y. Life cycle assessment of a steam thermolysis process to recover carbon fibers from carbon fiber-reinforced polymer waste. Int. J. Life Cycle Assess. 2018, 23, 1825-1838. [CrossRef]

57. Bertolini, M.; Bottani, E.; Vignali, G.; Volpi, A. Comparative Life Cycle Assessment of Packaging Systems for Extended Shelf Life Milk. Packag. Technol. Sci. 2016, 29, 525-546. [CrossRef]

58. Aryan, V.; Font-Brucart, M.; Maga, D. A comparative life cycle assessment of end-of-life treatment pathways for photovoltaic backsheets. Prog. Photovolt. Res. Appl. 2018, 26, 443-459. [CrossRef]

59. Haylock, R.; Rosentrater, K.A. Cradle-to-Grave Life Cycle Assessment and Techno-Economic Analysis of Polylactic Acid Composites with Traditional and Bio-Based Fillers. J. Polym. Environ. 2018, 26, 1484-1503. [CrossRef]

60. Oliveira, M.C.; Magrini, A. Life cycle assessment of lubricant oil plastic containers in Brazil. Sustainability 2017, 9, 576. [CrossRef]

61. Mendes Campolina, J.; São Leandro Sigrist, C.; Faulstich de Paiva, J.M.; Oliveira Nunes, A.; da Silva Moris, V.A. A study on the environmental aspects of WEEE plastic recycling in a Brazilian company. Int. J. Life Cycle Assess. 2017, 22, 1957-1968. [CrossRef]

62. De Meester, S.; Nachtergaele, P.; Debaveye, S.; Vos, P.; Dewulf, J. Using material flow analysis and life cycle assessment in decision support: A case study on WEEE valorization in Belgium. Resour. Conserv. Recycl. 2019, 142, 1-9. [CrossRef]

63. Sommerhuber, P.F.; Wenker, J.L.; Rüter, S.; Krause, A. Life cycle assessment of wood-plastic composites: Analysing alternative materials and identifying an environmental sound end-of-life option. Resour. Conserv. Recycl. 2017, 117, 235-248. [CrossRef]

64. Sun, X.; Liu, J.; Lu, B.; Zhang, P.; Zhao, M. Life cycle assessment-based selection of a sustainable lightweight automotive engine hood design. Int. J. Life Cycle Assess. 2017, 22, 1373-1383. [CrossRef]

65. Ferreira, S.; Cabral, M.; De Jaeger, S.; Da Cruz, N.F.; Simões, P.; Marques, R.C. Life cycle assessment and valuation of the packaging waste recycling system in Belgium. J. Mater. Cycles Waste Manag. 2017, 19, 144-154. [CrossRef]

66. Gu, F.; Guo, J.; Zhang, W.; Summers, P.A.; Hall, P. From waste plastics to industrial raw materials: A life cycle assessment of mechanical plastic recycling practice based on a real-world case study. Sci. Total Environ. 2017, 601-602, 1192-1207. [CrossRef] 
67. Toniolo, S.; Mazzi, A.; Pieretto, C.; Scipioni, A. Allocation strategies in comparative life cycle assessment for recycling: Considerations from case studies. Resour. Conserv. Recycl. 2017, 117, 249-261. [CrossRef]

68. Faraca, G.; Martinez-Sanchez, V.; Astrup, T.F. Environmental life cycle cost assessment: Recycling of hard plastic waste collected at Danish recycling centres. Resour. Conserv. Recycl. 2019, 143, 299-309. [CrossRef]

69. Vidal, R.; Moliner, E.; Martin, P.P.; Fita, S.; Wonneberger, M.; Verdejo, E.; Vanfleteren, F.; Lapeña, N.; González, A. Life Cycle Assessment of Novel Aircraft Interior Panels Made from Renewable or Recyclable Polymers with Natural Fiber Reinforcements and Non-Halogenated Flame Retardants. J. Ind. Ecol. 2018, 22, 132-144. [CrossRef]

70. Bataineh, K.M. Life-Cycle Assessment of Recycling Postconsumer High-Density Polyethylene and Polyethylene Terephthalate. Adv. Civ. Eng. 2020, 2020. [CrossRef]

71. Cosate de Andrade, M.F.; Souza, P.M.S.; Cavalett, O.; Morales, A.R. Life Cycle Assessment of Poly(Lactic Acid) (PLA): Comparison Between Chemical Recycling, Mechanical Recycling and Composting. J. Polym. Environ. 2016, 24, 372-384. [CrossRef]

72. Simon, B.; Amor, M.B.; Földényi, R. Life cycle impact assessment of beverage packaging systems: Focus on the collection of post-consumer bottles. J. Clean. Prod. 2016, 112, 238-248. [CrossRef]

73. Beigbeder, J.; Soccalingame, L.; Perrin, D.; Bénézet, J.C.; Bergeret, A. How to manage biocomposites wastes end of life? A life cycle assessment approach (LCA) focused on polypropylene (PP)/wood flour and polylactic acid (PLA)/flax fibres biocomposites. Waste Manag. 2019, 83, 184-193. [CrossRef]

74. Gear, M.; Sadhukhan, J.; Thorpe, R.; Clift, R.; Seville, J.; Keast, M. A life cycle assessment data analysis toolkit for the design of novel processes-A case study for a thermal cracking process for mixed plastic waste. J. Clean. Prod. 2018, 180, 735-747. [CrossRef]

75. Isola, C.; Sieverding, H.L.; Raghunathan, R.; Sibi, M.P.; Webster, D.C.; Sivaguru, J.; Stone, J.J. Life cycle assessment of photodegradable polymeric material derived from renewable bioresources. J. Clean. Prod. 2017, 142, 2935-2944. [CrossRef]

76. Hou, P.; Xu, Y.; Taiebat, M.; Lastoskie, C.; Miller, S.A.; Xu, M. Life cycle assessment of end-of-life treatments for plastic film waste. J. Clean. Prod. 2018, 201, 1052-1060. [CrossRef]

77. Demetrious, A.; Crossin, E. Life cycle assessment of paper and plastic packaging waste in landfill, incineration, and gasificationpyrolysis. J. Mater. Cycles Waste Manag. 2019, 21, 850-860. [CrossRef]

78. Santos, A.; Barbosa-Póvoa, A.; Carvalho, A. Life cycle assessment in chemical industry-A review. Curr. Opin. Chem. Eng. 2019, 26, 139-147. [CrossRef]

79. Plevin, R.J.; Delucchi, M.A.; Creutzig, F. Using Attributional Life Cycle Assessment to Estimate Climate-Change Mitigation Benefits Misleads Policy Makers. J. Ind. Ecol. 2014, 18, 73-83. [CrossRef]

80. Jambeck, J.R.; Geyer, R.; Wilcox, C.; Siegler, T.R.; Perryman, M.; Andrady, A.; Narayan, R.; Law, K.L. Plastic waste inputs from land into the ocean. Science 2015, 347, 768-771. [CrossRef] [PubMed]

81. Ghorbani, A.; Dechesne, F.; Dignum, V.; Jonker, C. Enhancing ABM into an Inevitable Tool for Policy Analysis. J. Policy Complex Syst. 2014, 1, 61-76. [CrossRef]

82. Onat, N.C.; Kucukvar, M.; Tatari, O.; Egilmez, G. Integration of system dynamics approach toward deepening and broadening the life cycle sustainability assessment framework: A case for electric vehicles. Int. J. Life Cycle Assess. 2016, 21, 1009-1034. [CrossRef]

83. Pinto, J.T.M.; Sverdrup, H.U.; Diemer, A. Integrating life cycle analysis into system dynamics: The case of steel in Europe. Environ. Syst. Res. 2019, 8. [CrossRef]

84. Wu, S.R.; Li, X.; Apul, D.; Breeze, V.; Tang, Y.; Fan, Y.; Chen, J. Agent-Based Modeling of Temporal and Spatial Dynamics in Life Cycle Sustainability Assessment. J. Ind. Ecol. 2017, 21, 1507-1521. [CrossRef]

85. Kwakkel, J.H. The Exploratory Modeling Workbench: An open source toolkit for exploratory modeling, scenario discovery, and (multi-objective) robust decision-making. Environ. Model. Softw. 2017, 96, 239-250. [CrossRef]

86. Zamagni, A. Life cycle sustainability assessment. Int. J. Life Cycle Assess. 2012, 17, 373-376. [CrossRef] 